\title{
Strategies for Gait Retraining in a Collegiate Runner with Transfemoral Amputation: A Case Report
}

\author{
Jillian Santer, PT, DPT ${ }^{1}$, Stephanie MacDonald, DO ${ }^{1}$, Katherine Rizzone, MD, MPH${ }^{1}$, Shawn Biehler, MS, CPO ${ }^{1}$, Tanya \\ Beiswenger, PT, SCS, ATC, MPH \\ 1 University of Rochester Medical Center \\ Keywords: movement system, track and field, collegiate athlete, adaptive athlete, phantom limb pain, amputee, transfemoral \\ https://doi.org/10.26603/001c.23671
}

\section{International Journal of Sports Physical Therapy}

Vol. 16, Issue 3, 2021

\section{Background}

More than fifty percent of people with limb amputations participate in sports or physical activity following amputation. Athletes with limb amputations may face additional challenges including phantom limb pain (PLP), psychological barriers, prosthetic complications, and gait abnormalities. Prevalence of PLP in the general amputee population is estimated to be as high as $85 \%$. Despite the high prevalence of PLP, there is little research regarding the use of gait training as a treatment for PLP among both the general amputee population and athletes.

\section{Case Description}

A 20-year old female collegiate track and field athlete presented with phantom knee pain brought on with running. The athlete demonstrated deficits in core and hip strength as well as decreased single leg stability bilaterally. Running gait analysis revealed circumduction with the prosthesis for limb advancement and increased vaulting with push off on the sound (uninvolved) limb. Gait retraining strategies were implemented to address video analysis findings and create a more efficient running gait and address phantom limb pain symptoms.

\section{Outcomes}

Rehabilitation and gait retraining strategies were effective in improving several clinical and functional outcomes in this case. Significant improvements were noted in PLP, running gait mechanics, and the patient's psychological and functional status as measured with a standardized outcome tool, the Patient-Reported Outcomes Measurement Information System ${ }^{\circledR}$ (PROMIS $^{\circledR}$ ).

\section{Discussion}

Running gait training following amputation could be a crucial component of rehabilitation for athletes in an attempt to lessen pain while running, especially in those experiencing phantom limb pain (PLP). Utilization of a multidisciplinary team in the gait retraining process is recommended. There is a need for further research to determine the effects of running gait retraining for management of PLP in athletes with amputation.

\section{Level of Evidence}

\footnotetext{
a Corresponding Author: Jillian Santer PT, DPT

University of Rochester Medical Center

Department of Orthopaedics and Physical Performance

Sports and Spine Rehabilitation

4901 Lac De Ville Boulevard

Building D, Suite 110

Rochester, NY 14618

Jillian_santer@urmc.rochester.edu
} 


\section{INTRODUCTION}

More than fifty percent of people with limb amputations participate in sports or physical activity following amputation. Exercise has been shown to positively influence selfesteem, perceived body image and better control of physical function in this population. ${ }^{1}$ Unfortunately, for many individuals, participation in sports may decrease following amputation due prosthetic barriers, including poor prosthetic fit and the prohibitive expense of a sports prosthesis. ${ }^{1}$

Additionally, athletes with amputations may experience pain resulting from the compensatory and asymmetrical movements that frequently occur following amputation. Older age, more proximal amputation and vascular changes are associated with impairments in activities of daily living and are likely obstacles to athletic endeavors. ${ }^{1}$ Athletes with amputations face further challenges when returning to activity including phantom limb pain, psychological barriers, prosthetic complications, and gait abnormalities.

Phantom limb pain (PLP) is defined as pain perceived as originating from the absent limb. Onset may occur soon after amputation or years later, although the majority of individuals with amputation report onset of symptoms within one year of amputation with reduced prevalence thereafter. $^{2}$ In a study of adults with a history of upper extremity amputations, two typical peak periods of onset were noted: one month after amputation and one year after amputation. ${ }^{3}$ The experience of PLP is variable and the etiology is not well understood. Proposed causes include altered peripheral neural mechanisms (including peripheral nerve irritation or neuroma), central neural mechanisms, or psychogenic factors including depression, anxiety, and altered body perception. ${ }^{24}$ Risk factors for the development of PLP include pain in the affected limb prior to amputation, pain in the intact residual limb, upper extremity amputation, and female gender. Prevalence of PLP in the general amputee population is estimated to be as high as $85 \% .^{5}$ There are no published data on the incidence of phantom limb pain in athletes with amputations.

Despite the high prevalence of PLP, there is little research regarding the use of gait training programs as a treatment for PLP among both the general amputee population and athletes. One systematic review evaluated gait training interventions post-amputation but did not address outcomes regarding PLP nor did it include athletes. ${ }^{6}$ While gait training with a prosthetic limb is an important component of rehabilitation following lower extremity amputation, it need not end once functional community ambulation has been achieved. For runners and other athletes with amputations, gait training can be a key aspect of rehabilitation to facilitate safe mechanics during higher levels of physical activity such as running and sports. Gait retraining may also have the potential to reduce phantom limb pain in lower extremity amputees through improved gait mechanics.

The purpose of this case report is to describe the use of running gait retraining strategies to address phantom limb pain in a collegiate athlete with transfemoral amputation. Due to a paucity of literature on this topic, when presented with the opportunity to provide gait training for one such transfemoral amputee collegiate sprinter it was necessary to develop a novel program utilizing a multidisciplinary approach.

\section{METHODS}

\section{CASE SUBJECT}

A 20-year old female National Collegiate Athletic Association (NCAA) Division III collegiate track and field athlete presented to her certified athletic trainer with phantom knee pain. Her medical history included right transfemoral amputation four years prior due to tibial osteosarcoma. At the initial presentation, the athlete reported two weeks of increased phantom knee pain with running, stair negotiation, and walking uphill. The athlete reported that she first noticed the pain one year ago, but it was now more persistent despite no new injuries or significant changes in training. Pain was reported to begin a few minutes into a running workout and continued throughout training and post-workout. The athlete experienced minimal to no pain at rest. In addition to pain, the athlete also expressed concern regarding the efficiency of her running gait.

After evaluation by her team physician, the athlete was referred to physical medicine and rehabilitation for physician consultation regarding phantom limb pain. The physician report stated that her right transfemoral amputation was well-healed with no evidence of skin breakdown, erythema, or tenderness. There were no concerns about her mood or affect at that time, and her walking gait was described as a normal reciprocal gait with right transfemoral amputation. It was recommended that she start gabapentin 300 milligrams daily. There were no diagnostic imaging studies performed at that time. The athlete reported no previous or concurrent rehabilitation for management of PLP, including gait retraining.

Prior to amputation, the athlete had participated on her high school cross country and track and field teams, focusing primarily on distance events. Post-amputation, she had not received any formal gait training to facilitate return to running. Since returning to running, the athlete had competed mostly in sprint events, although she reported being able to run comfortably at only one speed regardless of event or distance.

The athlete presented to her physical therapy evaluation wearing her walking prosthesis, with her running blade prosthesis in hand. She reported actively participating in preseason collegiate track workouts but was limiting her running activity due to PLP symptoms. The athlete reported no complaints or pathology of her sound limb. Her goals included being able to run comfortably without pain and to eventually complete a $5 \mathrm{k}$.

\section{PROSTHESIS DESIGN}

The subject of this case report utilizes a socket design which focuses on maximum comfort and control, all the while achieving a light-weight solution. Suction suspension minimizes in socket movements that may trigger undesired pain and skin breakdown. These characteristics are imperative for a highly active individual with increased demands. Thin socks were also implemented to maximize suction and minimize energy loss. The athlete's prosthetic knee (Cheetah 
Knee by Ossur, headquartered in Reykjavík, Iceland) (Figure $\underline{1 \mathrm{a} \text { and } 1 \mathrm{~b}}$ ) is an ideal choice for running and sprinting activities. The polycentric design promotes stability in stance, but allows controlled deceleration during athletic activities. Multiple aspects of swing can be adjusted independently to yield the most energy and time efficient outcomes. The athlete's prosthetic foot (Flex Run by Ossur) leverages optimal prosthetic ingenuity with Nike's traction and sole technologies. The foot does not require a sneaker, and the stiffness and design varies according to an athlete's weight in order to facilitate an optimal energy storage and return. When ordering this type of prosthetic foot, one must consider the loads placed on it (a function of patient weight) and the frequency (a function of velocity) in which it will compress. Loads and frequencies change between running and ambulation, and therefore when categorized for running, such a foot would not be optimal for everyday ambulation. ${ }^{7}$

\section{PHYSICAL THERAPY EVALUATION}

Initial evaluation by a physical therapist specializing in running medicine revealed hip extension strength deficits bilaterally, with the involved right leg exhibiting 4-/5 strength and sound left limb with $4 / 5$ strength. Hip abduction muscle strength was $4 / 5$ bilaterally. Sound limb plantarflexion and inversion strength were also $4 / 5$. Standard core strength measurements were unable to be assessed due to the amputation, however the athlete was observed to demonstrate decreased core stability as evidenced by increased anterior tilt and lumbar lordosis during performance of supine and standing core exercises. The athlete demonstrated knee valgus and pronation on the sound limb with single leg squatting, and decreased stability with single leg balance bilaterally. She was unable to balance unilaterally on her prosthetic side, in either her walking or running prosthesis.

A 2-D video analysis of the athlete's running gait with her running blade prosthesis was taken at the initial physical therapy visit. Due to reported discomfort when running on a treadmill, videos were taken outdoors over a series of five 75-meter trials. Initial findings of the running gait analysis revealed circumduction with the prosthesis for limb advancement and increased vaulting with push off on the sound limb. During swing phase relative to the prosthesis, the athlete demonstrated increased abduction as well as ipsilateral trunk lean at mid swing, followed by significant hip flexion and internal rotation to further advance the prosthesis in preparation for initial contact. Decreased knee flexion with the prosthesis was noted throughout swing phase primarily due to limb circumduction momentum. The athlete utilized a slight posterior trunk lean during initial contact and midstance on the prosthetic side, versus an upright posture with initial contact and midstance on the sound limb. Increased lumbar lordosis, knee valgus and pronation were noted during midstance on the sound limb. The athlete demonstrated a forefoot strike pattern on the sound limb. There was evidence of overstriding bilaterally in her video gait analysis.
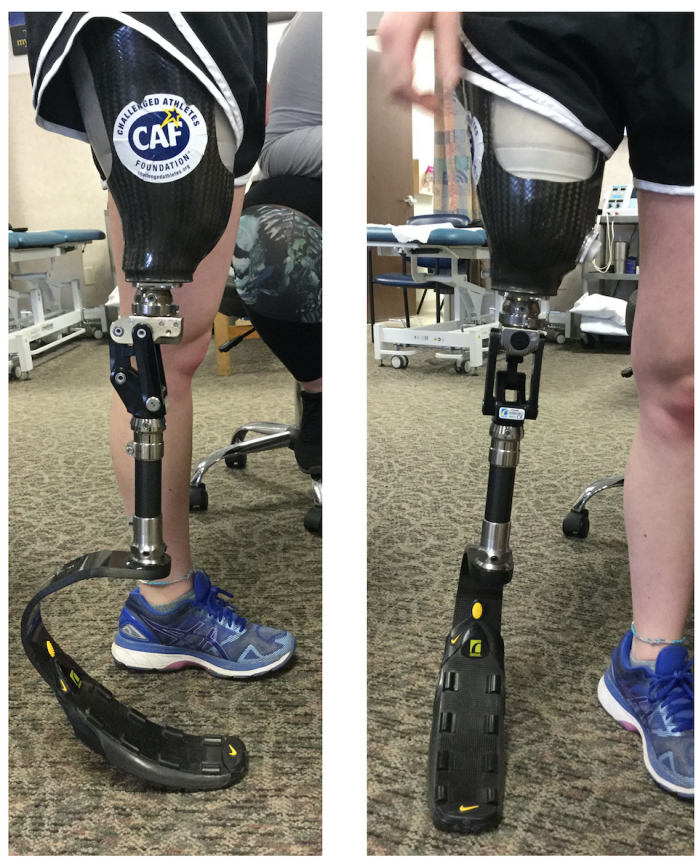
Figure 1: Static alignment of prosthesis with patient
loading sound and involved sides equally

\section{INTERVENTION}

Early intervention consisted of core and hip strengthening exercises bilaterally, focusing on pelvic stability during reciprocal leg movement. Sound limb strengthening was also implemented to address dynamic valgus and pronation tendencies noted during both the initial examination in standing and video gait analysis with running. Dynamic exercises were performed to improve balance and strength while weight shifting on and off the prosthetic side during stepping movements. This was initiated in the athlete's walking prosthesis and progressed to the running blade prosthesis, utilizing multiplanar and multi-height stepping and lunging exercises. Single leg balance exercises were introduced bilaterally as well. Ultimately, exercises focusing on turnover, speed and power were implemented (Table 1). Throughout the course of rehabilitation, exercises in these categories were progressed both in the clinic and for the athlete's home program.

It was crucial that the athlete develop confidence in her use of the running blade prosthesis, as it differed greatly from the capabilities of her day-to-day prosthesis. One strategy to increase confidence was named "fast feet" and consisted of quickly shifting weight from one leg to another. This required the athlete to accept weight onto the prosthetic side, then push off to land back on the sound limb without any forward motion initially. Speed, time spent on each limb and base of support were variables that were manipulated over time to increase challenge as the athlete's skill and capacity progressed. This activity required the athlete's weight to be balanced on her forefeet. As the video gait analysis revealed, the athlete already employed a forefoot strike pattern on her sound limb while running. This 
Table 1: Exercise Interventions and Progressions

\begin{tabular}{|l|l|}
\hline Core activation & $\begin{array}{l}\text { Progression: } \\
\text { Single leg bridging with foam roller } \\
\text { Standing bird dog } \\
\text { Side planks } \\
\text { Suspension trainer (TRX) knee drives }\end{array}$ \\
\hline Weight Acceptance & $\begin{array}{l}\text { Progression: } \\
\text { Double leg squatting } \\
\text { Focus: Increase confidence with weight bearing and stability on } \\
\text { running prosthesis }\end{array}$ \\
& $\begin{array}{l}\text { Multi-directional lunging } \\
\text { Single leg balance }\end{array}$ \\
& $\begin{array}{l}\text { Standing single leg weight shifts } \\
\text { Wall lean knee drive }\end{array}$ \\
\hline Strength & $\begin{array}{l}\text { Single leg heel raises } \\
\text { Lateral step downs } \\
\text { Lateral lunges }\end{array}$ \\
Focus: Sound limb dynamic stability & Single leg hip hinge progression \\
\hline Speed & Fast feet \\
Focus: Encourage quick turnover, forefoot weight bearing and & Agility ladder \\
decreased stance time & Rear kicks \\
\hline
\end{tabular}

was beneficial, as the prosthetic foot requires a forefoot strike pattern with running. 8,9 The prescribed "fast feet" exercise enabled her to build strength, balance and assurance while transferring and maintaining weight onto the forefoot of her running blade prosthesis.

The initial goal of running gait modification was to decrease the stride length and vaulting noted on video analysis, as the working theory was that the excessive impact sustained while landing on the prosthetic limb was contributing to the athlete's symptoms. To address this, forward motion was added to the "fast feet" exercise, starting with a wide base of support. This helped the athlete learn to push off her sound limb and land on her prosthetic leg quickly. Progression included increasing speed and narrowing base of support until the athlete began to run more naturally. Through this strategy, stride length decreased and the vaulting seen on initial gait analysis was minimized, although a circumduction pattern for limb advancement remained. The athlete did not experience pain with these drills in clinic, during her home exercise program, or during track practice sessions.

Given that her prosthesis included a hydraulic knee, it was desirable to build a more efficient running gait pattern in which her knee flexed and extended in the sagittal plane, rather than the circumduction pattern that had been observed. A forefoot strike pattern assisted with this, as pressure on the forefoot combined with a forward lean is required to facilitate prosthetic knee flexion.9,10 Static and dynamic exercises emphasizing controlled hip flexion and knee drive were introduced to facilitate this mechanism. These aimed to help the athlete increase balance, strength and confidence in weight bearing through the toe of her prosthesis in order to facilitate the transition to knee flexion during running to produce a more natural and efficient gait pattern. ${ }^{11}$ As the athlete became more comfortable with this running pattern, additional running drills were added to develop her ability to run at varying speeds.

As the athlete progressed to running at different speeds, stride length changes were noted. A return of increased stride length when sprinting was noted bilaterally. While this may have initially been due to fear of prosthetic swing clearance, it was only exhibited during sprinting and therefore might simply represent a functional adaptation for generating speed and power. Further observation demonstrated that the athlete maintained knee flexion through swing phase on the prosthetic side despite this change in stride length. There was no degradation of her newly developed knee flexion and she was able to sprint without vaulting and with equal stride length bilaterally.

The athlete's athletic trainer reported that she continued to work with the athlete on occasion as well providing heat, ice, and electrical stimulation in the training room. The remainder of the athlete's rehabilitation exercises were performed on the athlete's own time and with physical therapy. The athlete did participate in indoor and outdoor track workouts with her collegiate team throughout the course of her rehabilitation.

\section{OUTCOMES}

Over the course of rehabilitation, the athlete in this case demonstrated significant improvements in several clinical and functional outcomes, including reported PLP symptoms, running gait pattern, psychological measures, and overall function. The athlete had complete resolution of her phantom limb pain after eight weeks of rehabilitation. As a result, she did not initiate the prescribed medication and did not require additional follow up from physiatry. There were no adverse events or skin breakdown during the course of physical therapy.

Running gait pattern improvements were measured via $2 \mathrm{D}$ video gait analysis. Running gait analysis was performed at the first visit and approximately every two weeks throughout the first four months of rehabilitation. Kinematic video and still-frame analysis of key phases of the gait cycle demonstrated post-treatment improvements in limb circumduction, stride length, and vertical displacement. Using 2-D analysis, marked improvements in cadence, trunk 
Table 2: Gait Analysis Changes

\begin{tabular}{|c|c|c|c|}
\hline Time of Treatment & \multicolumn{3}{|l|}{2 months } \\
\hline Therapy Sessions & \multicolumn{3}{|l|}{6 visits } \\
\hline Changes in Gait Pattern & Initial & End & Difference \\
\hline Cadence & 140 spm & $192 \mathrm{spm}$ & $52 \mathrm{spm}$ \\
\hline Forward Trunk Lean (midstance) & $5^{\circ}$ & $12^{\circ}$ & $7^{\circ}$ \\
\hline Peak Hip Abduction (prosthesis, swing phase) & $11.0^{\circ} \mathrm{abd}$ & $11.1^{\circ}$ add & $22.1^{\circ} \mathrm{deg}$ \\
\hline
\end{tabular}

Spm= steps per minute, abd= abduction, add= adduction

Table 3: PROMIS ${ }^{\circledR}$ Scores

\begin{tabular}{|l|l|l|l|}
\hline Time of Treatment & 7 months \\
\hline Therapy Sessions & 16 visits & $\begin{array}{l}\text { Difference } \\
\text { Delta (SD) }\end{array}$ \\
\hline PROMIS $^{\circledR}$ Scores & $\begin{array}{l}\text { Initial } \\
\text { (T-score) }\end{array}$ & $\begin{array}{l}\text { End } \\
\text { (T-score) }\end{array}$ & $4(.4)$ \\
\hline Adult Physical Function & 50 & 54 & $13(1.3)$ \\
\hline Adult Pain Interference & 52 & 39 & $5(.5)$ \\
\hline Adult Depression & 39 & 34 & \\
\hline
\end{tabular}

Note: In most cases across most domains, a PROMIS ${ }^{\circledast}$ score of 50 represents the mean in the U.S. general population and a standard deviation is described as a 10 point change. $(22)$

lean and peak abduction of the athlete's prosthesis were noted. (Table 2) Following her gait retraining interventions, the athlete demonstrated increased hip adduction and reduced hip flexion and internal rotation during swing phase with her prosthesis. The athlete also demonstrated improvement in single leg stability on her sound limb, with decreased valgus and lateral trunk lean noted in midstance (Table 2). After the completion of her rehabilitation, the athlete was able to run at varying speeds as required to train and compete in track events. The athlete reported significant improvement in running efficiency after the therapy and gait interventions. Over the course of her subsequent indoor track season, the athlete also reported objective, functional success in the form of a three second reduction in her 60-meter dash time, with a personal best recorded at 14.64 seconds.

Physical and psychological outcomes were assessed using the Patient-Reported Outcomes Measurement Information System ${ }^{\circledR}$ (PROMIS $^{\circledR}$ ). The PROMIS ${ }^{\circledR}$ computer adaptive testing instruments are validated, publicly available, NIHdeveloped instruments that determine standard scores of various self-reported patient outcomes such as physical function, depression, and pain interference. ${ }^{12}$ Over seven months totaling 16 visits, the subject of this case report demonstrated improvement in self-reported PROMIS ${ }^{\circledR}$ scores across three domains: Adult Physical Function, Adult Pain Interference, and Adult Depression, with the largest improvement noted in Adult Pain Interference (1.3 SD). (Table 3)

\section{DISCUSSION/CONCLUSION}

Running gait training following amputation could be a crucial component of rehabilitation for athletes in an attempt to lessen pain while running, especially in those experiencing phantom limb pain (PLP). Currently, the authors are unaware of any literature regarding gait retraining to address phantom limb pain in amputee runners. It was unclear what led to the development of phantom limb pain in this particular athlete, though in general the development of PLP is not fully understood. The authors suspect that the cause of PLP in this case was multifactorial. Because the PLP occurred mostly with running and not at rest, it was hypothesized that inefficient running gait was a key contributing factor.

A few potential mechanisms for PLP are described in the literature. ${ }^{2,13}$ One potential cause of PLP is explained by a peripheral nerve irritation hypothesis. In this pathway, mechanical irritation of the distal residual limb generates PLP via noxious stimulation of a peripheral nerve. This explanation may be applicable in this case, as we do believe that the athlete's inefficient running gait pattern could have contributed to abnormal stress on her distal residual limb. In this case, PLP improved after a rehabilitation program that included running gait interventions which has the potential to decrease peripheral nerve irritation of the distal residual limb.

A few variables within a running prosthesis which can adversely affect athletic performance include suboptimal socket suspension ${ }^{10}$ and componentry malalignments. ${ }^{14,15}$ The athlete in this case did experience prosthesis fit difficulties towards the end of her physical therapy intervention, reporting loosening of the socket when running and 
the feeling that her prosthesis was "falling off." This loosening was observed to create increased internal rotation of the prosthesis with hip flexion while running and overall reduced running speed. While the literature does describe dissatisfaction with prosthesis as a possible contributing factor to PLP, ${ }^{5}$ in this case the athlete's symptoms had resolved prior to the onset of fit issues and resulting prosthesis modifications. Modifications to the socket were made by the athlete's prosthetist and thin socks were provided to help accommodate limb volume fluctuation, ultimately providing a better fit. The athlete's symptoms did not return throughout her prosthesis adjustment period. While the fit of the running prosthesis was not believed to be a factor in the onset of PLP in this case, additional study of a larger cohort would be required to determine if these variables might explain PLP in other transfemoral amputee athletes, with or without competitive running aspirations.

Changes in physical function, pain and mood were assessed through a self-reported outcome measurement $\left(\mathrm{PROMIS}^{\circledR}\right.$ ), where a t-score of 50 is indicative of the general US population within each domain. Age related norms have also been established for each of the listed domains; for age range 18-34 years norms are as follows: Adult Physical Function: 55.1 (SD 8.4) Adult Pain Interference 47.8 (SD 9.0) and Adult Depression: 52.3 (SD 10.9). ${ }^{16}$ The authors are unaware of established norms related to individuals with amputation; further study into this domain could be indicated. The case athlete demonstrated the most improvement in pain interference, with a final t-score of 39. Remarkably, the subject concluded her treatment with a t-score less than both the general population and her peers. She also reported a final t-score of 54 in the Adult Physical Function domain, equal to both general and age-related norms in the United States population.

The positive benefits of activity on mood and psychological health are well known, ${ }^{17}$ but the effects were not able to be specifically delineated in this single case. The athlete did demonstrate improvement in PROMIS ${ }^{\circledR}$ score in the depression domain, however this did not achieve the suggested minimal clinical important difference (MCID) of greater than .5 of the standard deviation. ${ }^{18}$ (Table 3). It is noted that the athlete's beginning and end PROMIS ${ }^{\circledR}$ scores for Adult Depression were below the average and age-related population norms, potentially indicating better mood compared to general population and/or peers. While the current case did not yield enough data to draw definitive conclusions about changes in depression, this could be an interesting area of future study should this area of rehabilitation be expanded to a larger population.

A limitation of this case report was the use of a $2 \mathrm{D}$ video analysis system rather than the $3 \mathrm{D}$ video analysis which is typically considered the gold standard. However, the use of a $2 \mathrm{D}$ system can be ideal in a clinical setting and is often a more practical and cost efficient tool for the rehabilitation clinician. Measurements of trunk lean and peak hip abduction were taken on a still frame, and are therefore not as accurate as compared to 3D measures. Despite the limitations of 2D data capture, there were significant clinical changes in peak hip abduction based on degree of change and visual observation (Figure 2). Changes described in this study were consistently noted at the same phase of running respec-
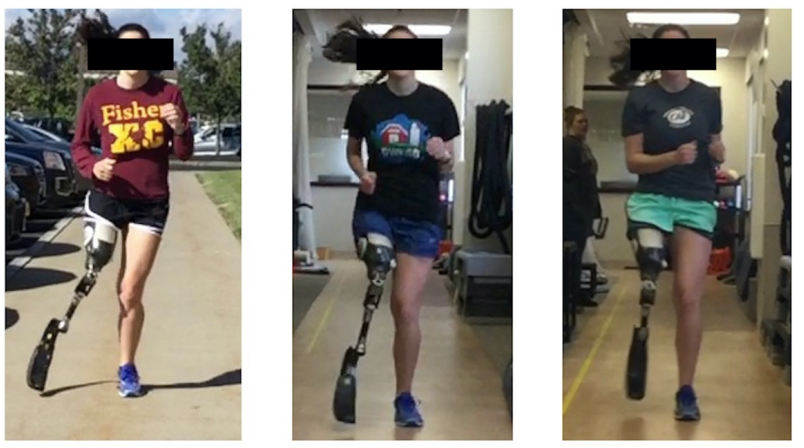

Figure 2: Midstance on the athletes sound limb noted on Day 1: Initial Evaluation (left); at one month (middle); and at two months (right). Changes in alignment of both the prosthesis and sound limb can be appreciated at this phase of running gait.

tively and occurred over multiple strides of each recorded run.

Improvement in core strength and stability were an imperative part of this athlete's rehabilitation, as it was necessary to increase pelvic control to promote ideal trunk position with running. While traditional core strength measures have been described and utilized in able- bodied athletes, the authors are unaware of the validity of these same tests in the case of an athlete following amputation. Further investigation into the validity of these tests for the amputee population should be completed in the future.

Future investigations could explore gait retraining techniques used in this case toward the development of a program that could be applied to other transfemoral or transtibial amputee athletes. Other opportunities for study include examination of factors associated with the development of PLP in runners to better understand if there are risks for development of PLP unique to the amputee athlete population versus the general amputee population, or if there are actually other protective factors at work. Additional study of a larger cohort would be required to determine if the factors and interventions illustrated in this case might respectively explain or treat PLP in other transfemoral amputee athletes, with or without competitive running aspirations. Since PLP in general is poorly understood it is important to keep in mind that rehabilitation programs may vary in an attempt to individualize treatment approaches for a wide variety of needs until a greater understanding of key variables and treatment pathways emerge.

\section{CONCLUSIONS}

This case report summarizes the successful evaluation and treatment of a female collegiate running athlete with transfemoral amputation that presented with PLP and running gait impairment. Key principles in the case of this athlete include diagnosis of running gait impairment by a physical therapist specializing in running medicine as well as a unique rehabilitation program designed by this therapist to address the gait abnormalities that we believe significantly 
contributed to the resolution of her PLP. Over the course of her treatment, this athlete worked with a multidisciplinary team including team athletic trainer, team physician, physical therapist, prosthetist and physiatrist. The unique expertise of each of the providers discussed in this report was beneficial to the care of this athlete and is a good model going forward for the collaboration that is necessary for a successful outcome in this type of case.

\section{CONFLICTS OF INTEREST}

The authors of this paper have nothing to disclose.

Submitted: June 10, 2020 CDT, Accepted: November 26, 2020 CDT 


\section{REFERENCES}

1. Bragaru M, Dekker R, Geertzen JHB, Dijkstra PU. Amputees and sports: A systematic review. Sports Med. 2011;41(9):721-740. doi:10.2165/11590420-0000 $\underline{00000-00000}$

2. Fuchs X, Flor H, Bekrater-Bodmann R. Psychological factors associated with phantom limb pain: A review of recent findings. Pain Res Manag. 2018;2018:5080123. doi:10.1155/2018/5080123

3. Schley MT, Wilms P, Toepfner S, et al. Painful and nonpainful phantom and stump sensations in acute traumatic amputees. J Trauma. 2008;65(4):858-864. d oi:10.1097/ta.0b013e31812eed9e

4. Bunch WH. Management of pain in the amputee. Chapter 27. In: Bowker HK, Michael JW, eds. Atlas of Limb Prosthetics: Surgical, Prosthetic, and Rehabilitation Principles. Mosby; 1992.

5. Desmond D, Gallagher P, Henderson-Slater D, Chatfield R. Pain and psychosocial adjustment to lower limb amputation amongst prosthesis users. Prosthet Orthot Int. 2008;32(2):244-252. doi:10.1080/0 $\underline{3093640802067046}$

6. Highsmith MJ, Andrews CR, Millman C, et al. Gait training interventions for lower extremity amputees: A systematic literature review. Technol Innov. 2016;18(2-3):99-113. doi:10.21300/18.2-3.2016.99

7. Noroozi S, Ong ZC, Khoo SY, Aslani N, Sewell P. Dynamic characterisation of Össur Flex-Run prosthetic feet for a more informed prescription. Prosthet Orthot Int. 2019;43(1):62-70. doi:10.1177/030 $\underline{9364618789449}$

8. Nolan L. Carbon fibre prostheses and running in amputees: A review. Foot Ankle Surg. 2008;14(3):125-129. doi:10.1016/j.fas.2008.05.007

9. Diebal-Lee AR, Kuenzi RS, Rábago CA. Return to running following a knee disarticulation amputation: A case report. Int J Sports Phys Ther. 2017;12(4):655-669.

10. Sano Y, Makimoto A, Hashizume S, et al. Leg stiffness during sprinting in transfemoral amputees with running-specific prosthesis. Gait Posture. 2017;56:65-67. doi:10.1016/j.gaitpost.2017.04.038
11. Highsmith MJ, Kahle JT, Miro RM, Mengelkoch LJ. Bioenergetic differences during walking and running in transfemoral amputee runners using articulating and non-articulating knee prostheses. Technol Innov. 2016;18(2-3):159-165. doi:10.21300/18.2-3.2016.159

12. Broderick JE, Schneider S, Junghaenel DU, Schwartz JE, Stone AA. Validity and reliability of patient-reported outcomes measurement information system instruments in osteoarthritis. Arthritis Care Res. 2013;65:1625-1633. doi:10.1002/acr.22025

13. Subedi B, Grossberg GT. Phantom limb pain: Mechanisms and treatment approaches. Pain Res Treat. 2011;2011:864605. doi:10.1155/2011/864605

14. Groothuis A, Houdijk $\mathrm{H}$. The effect of prosthetic alignment on prosthetic and total leg stiffness while running with simulated running-specific prostheses. Front Sports Act Living. 2019;1(1):1-10. doi:10.3389/fs por.2019.00016

15. Schmalz T, Blumentritt S, Jarasch R. Energy expenditure and biomechanical characteristics of lower limb amputee gait: the influence of prosthetic alignment and different prosthetic components. Gait Posture. 2002;16(3):255-263. doi:10.1016/s0966-636 2(02)00008-5

16. Rose M, Bjorner JB, Gandek B, Bruce B, Fries JF, Ware JE Jr. The PROMIS physical function item bank was calibrated to a standardized metric and shown to improve measurement efficiency. J Clin Epidemiol. 2014;67(5):516-526. doi:10.1016/j.jclinepi.2013.10.02 $\underline{4}$

17. Sharma A, Madaan V, Petty FD. Exercise for mental health. Prim Care Companion J Clin Psychiatry. 2006;8(2):106. doi:10.4088/pcc.v08n0208a

18. Norman GR, Sloan JA, Wyrwich KW. Interpretation of changes in health-related quality of life: The remarkable universality of half a standard deviation. Med Care. 2003;41(5):582-592. doi:10.109 7/01.mlr.0000062554.74615.4c 